\section{Pleomorphic, Pleomorphism}

Carol L. Armstrong

Child and Adolescent Psychiatry and Behavioral

Sciences, The Children's Hospital of

Philadelphia, Philadelphia, PA, USA

\section{Synonyms}

Pleomorphic changes

\section{Definition}

In neoplasms, pleomorphisms are changes that occur to neurons involved in the neoplasm, causing great changes in the size and shape of the cell and its nucleus, and bizarre cytologic features. The tumors themselves may take on a variety of shapes and sizes and can be described as pleomorphic. These changes can make the cells difficult to differentiate on pathologic examination. In general, microglial pleomorphism is a basic response to injury in other settings. Pleomorphic changes are one of the criteria in the World Health Organization criteria for tumor grading and contribute to a diagnosis of increasing malignancy.

\section{Cross-References}

> Tumor Grade 